\title{
Pro-inflammatory effect of a traditional Chinese medicine formula with potent anti-cancer activity in vitro impedes tumor inhibitory potential in vivo
}

\author{
LEI XIA $^{1,2^{*}}$, MAKSYM PLACHYNTA ${ }^{2 *}$, TANGJINGJUN LIU $^{2}$, XIAO XIAO $^{2}$, JIALEI SONG $^{2}$, XIAOGANG LI $^{2}$, \\ MU ZHANG ${ }^{2}$, YAO YAO ${ }^{2}$, HENG LUO $^{2}$, XIAOJIANG HAO ${ }^{2,3}$ and YAACOV BEN-DAVID ${ }^{2,3}$ \\ ${ }^{1}$ School of Pharmaceutical Sciences, Guizhou University, Guiyang, Guizhou 550025; ${ }^{2}$ Division of Biology \\ and Chemistry, The Key Laboratory of Chemistry for Natural Products of Guizhou Province \\ and Chinese Academy of Sciences, Guiyang, Guizhou 550008; ${ }^{3}$ Department of Biological \\ Sciences, Guiyang Medical University, Guiyang, Guizhou 550025, P.R. China
}

Received May 5, 2016; Accepted September 9, 2016

DOI: $10.3892 / \mathrm{mco} .2016 .1059$

\begin{abstract}
Medicinal formulas are a part of the complex discipline of traditional Chinese medicine that has been used for centuries in China and East Asia. These formulas predominantly consist of the extracts isolated from herbal plants, animal parts and medicinal minerals. The present study aimed to investigate the impact of 150 formulas, used as non-prescription drugs in China, on the treatment of cancer. A formula was identified, C54, commonly used to treat sore throats, which exhibited marked growth inhibition in vitro, associated with cell cycle arrest and apoptosis. Cytotoxicity was, in part, due to the ability of C54 to inhibit the expression and function of the transcription factor, Fli-1, leading to marked inhibition of leukemic cell growth in tissue culture. However, when injected into a model of leukemia initiated by Fli-1 activation, C54 only exhibited a limited tumor inhibition. C54 also did not suppress xenograft growth of the breast cancer cell line, MDA-MB-231, orthopedically transplanted into the mammary fat pad of severe combined immunodeficiency (SCID) mice. Notably, splenomegaly and accumulation of inflammatory $\mathrm{CD} 11 \mathrm{~b}^{+} / \mathrm{Grl}^{+}$monocytes were observed in the tumors and spleens of C54-treated mice. As inflammation is known to accelerate tumor progression, this immune response may counteract the cell-autonomous effect
\end{abstract}

Correspondence to: Dr Yaacov Ben-David or Dr Xiaojiang Hao, Division of Biology and Chemistry, The Key Laboratory of Chemistry for Natural Products of Guizhou Province and Chinese Academy of Sciences, Province Science City, High Tech Zone, Baiyum, Guiyang, Guizhou 550008, P.R. China

E-mail: yaacovbendavid@hotmail.com

E-mail: haoxj@mail.kib.ac.cn

*Contributed equally

Key words: traditional Chinese medicine drugs, apoptosis, Fli-1, inflammation, cancer progression of C54, and account for its limited tumor inhibitory effect in vivo. Combining C54 with an anti-inflammatory drug may improve the potency of C54 for treatment of certain cancers. The present study has highlighted the complexity of Chinese medicinal compounds and the need to thoroughly analyze their systemic effects at high concentrations in vivo.

\section{Introduction}

Traditional Chinese medicine (TCM) has been used for over 2,000 years in China, and has recently become an accepted alternative therapy in Western countries. TCM is based on syndrome patterns that are diagnosed through complex symptoms, and with the goal of reaching a 'yin-yang' balance in a person's body prior to the onset of disease. TCM includes Chinese herbal medicine, remedial massage (termed 'An-Mo-Tui-Na'), diet, lifestyle advice, as well as acupuncture, exercise and breathing therapy (including qigong). In 1950, in the People's Republic of China, TCM was standardized, and nowadays is frequently administered through drug formulas and other means for the treatment of diverse diseases. The drug formulas predominantly are composed of extracts from medicinal plants, animal and human tissue, as well as from medicinal minerals (1). Certain of these components represent the principal agent in TCM, and others serve as an adjuvant to improve the effects of, or facilitate the delivery of, the active component.

TCM theory and practice are not based upon scientific knowledge, and its effectiveness is not well understood; neither has it been well researched (2). There are concerns over a number of potentially toxic plants, animal parts and mineral Chinese medicinal compounds $(1,3)$. Furthermore, due to the unknown interactions among the various ingredients of drug formulations and complex interactive biological systems, interpretation of the effects due to TCM becomes highly complicated. Thus, improved methods of evaluation in combination with randomized clinical trials are warranted to assure the effectiveness of TCM for various diseases.

Chinese medicinal philosophy believes that toxic heat is the root cause of cancer progression. Thus, numerous herbs 
used in TCM were developed to offset this toxic effect, and have been used for treatment of cancer. Previous studies have also suggested that the principal TCM effect may stem from its ability to alter a patient's immune system, leading to cancer inhibition $(4,5)$. The present study aimed to investigate the effectiveness of 150 drug formulations used in TCM for leukemia induction and progression. A drug formulation, designated C54, was identified that markedly inhibited leukemia cell proliferation through cell cycle arrest and apoptosis. This TCM drug was originally administered for the treatment of sore throats, although it has never previously been tested for cancer therapy. The present study also revealed that C54 inhibits the function of the oncogene, Fli-1, in leukemic cells. Notably, when C54 was administered in a mouse model of leukemia, the drug only moderately inhibited the induction and progression of leukemia. It was also revealed that this occurred, at least in part, through the induction of inflammation and increased infiltration of tumor inhibitory monocytes, processes that are known to accelerate cancer progression. Taken together, the present study has revealed the complexity of TCM, and the necessity to study the systemic effects of individual components of drug formulation when applying the identical methods to other diseases.

\section{Materials and methods}

Cell lines. The murine Friend virus-induced erythroleukemic cell line, CB7, human erythroleukemic cell lines, K562 and HEL, the human breast cancer cell line, MDA-MB-231, and the human melanoma (WM9) and HEK293T cell lines were maintained in Dulbecco's modified Eagle's medium supplemented with 5\% fetal bovine serum at $37^{\circ} \mathrm{C}$ (HyClone ${ }^{\mathrm{TM}} \mathrm{Cell}$ Culture; GE Healthcare, Sydney, NSW, Australia).

Tumor induction and in vivo drug studies. Viral supernatants from NIH-3T3 cells transduced with Friend murine leukemia virus (F-MuLV) clone 57 plasmid (6) were harvested and frozen at $-80^{\circ} \mathrm{C}$. Newborn BABL/c mice were inoculated with F-MuLV injections administered intraperitoneally (IP), as previously described (6). At 5 weeks post-infection, leukemic mice were injected IP every other day, for a total of six injections with either the C54 drug (50 mg/kg) or dimethylsulfoxide (DMSO) as a control, and monitored for any signs of disease. At the end point of the treatment, when the mice were exhibiting signs of morbidity from severe leukemia, the animals were sacrificed by cervical dislocation, and were used to determine the survival rate, spleen weight and hematocrit value. Hematocrit values were measured by tail blood collection in $200 \mathrm{ml}$ heparinized capillary tubes (Drummond Scientific, Broomall, PA, USA); the blood was centrifuged at $1,000 \times \mathrm{g}$ for $15 \mathrm{~min}$, and subsequently evaluated using a hematocrit gauge. Two groups of MDA-MB-231 cells $\left(1 \times 10^{6}\right)$ were injected into the mammary fat pad of anesthetized severe combined immunodeficiency (SCID) mice, as previously described (7). After tumors had reached $0.5 \mathrm{~cm}$ in diameter, mice were given a dose of $150 \mathrm{mg} / \mathrm{kg}$ drug via gavage, every day for 2 weeks. All animal studies were conducted in accordance with the ethical standards of China's animal care and use of laboratory animals. The study protocol was approved by the ethics committee on animal experiments of Guizhou Medical University.
Determination of the $I C_{50}$ values (i.e., the concentration of drug required to give half-maximal inhibition), cell cycle and apoptosis analysis. Triplicate cultures of the CB7, HEL, K562, WM9, MDA-MB-237 and PC3 cell lines were incubated with different concentrations of C54 or DMSO as a control for three days. Cell were subsequently subjected to an MTT assay by adding 3-(4,5-dimethylthiazol-2-yl)-2,5-diphenyltetrazolium bromide to the culture for $4 \mathrm{~h}$. Following removal of the supernatant, $200 \mathrm{ml}$ DMSO was added to dissolve the formazan crystals. The absorbance was read using a Synergy 2 microplate reader (BioTek Instruments, Inc., Winooski, VT, USA) at $490 \mathrm{~nm}$. For apoptosis and cell cycle analysis, CB7, HEL and $\mathrm{K} 562$ cell lines were incubated at $37^{\circ} \mathrm{C}$ with $\mathrm{C} 54$ or DMSO as a control for $24 \mathrm{~h}$; subsequently, the cells were washed in cold phosphate-buffered saline (PBS). For the apoptosis experiments, cells were stained using an annexin $\mathrm{V}$ and propidium iodide (PI) Apoptosis Detection kit (BD Biosciences, Franklin Lakes, NJ, USA), prior to flow cytometric analysis. For cell cycle analysis, cells were fixed in cold $75 \%$ ethanol overnight at $-20^{\circ} \mathrm{C}$. Following a further wash with cold PBS, cells were stained in PI for $40 \mathrm{~min}$ at $37^{\circ} \mathrm{C}$, and then subjected to flow cytometric analysis. $\mathrm{IC}_{50}$ values were calculated using appropriate software.

Flow cytometric analysis. Immunofluorescence staining was performed to determine the expression of Gr-1, Mac-1 (CD11b) and major histocompatibility complex class II (MHCII) molecules on the tumors and splenocytes of control (DMSO) and C54-treated mice, as described previously (8). In brief, $1 \times 10^{6}$ cells were incubated with anti-CD16/CD32 blocking antibody (cat. no. 14-0161-82; eBioscience, San Diego, CA, USA) for $10 \mathrm{~min}$ at $4^{\circ} \mathrm{C}$. Cells were stained with primary antibodies for $30 \mathrm{~min}$ on ice. The primary antibodies were as follows: Phycoerythrin-conjugated anti-mouse $\mathrm{Gr} 1$ (cat. no. 17-5931-81), Mac1 (cat. no. 17-0112-82) and MHCII (cat. no. 11-5322-82) antibodies (all from eBioscience). Cells were subsequently washed and resuspended in PI $(0.1 \mathrm{mg} / \mathrm{ml}$; Sigma-Aldrich, St. Louis, MO, USA) to exclude dead cells. A total of $10^{4}$ events were collected using a FACSCalibur ${ }^{\mathrm{TM}}$ flow cytometer and analyzed using CellQuest ${ }^{\mathrm{TM}}$ Pro software (both from BD Biosciences).

Western blot analysis. Western blot analysis was performed as previously described (6). Polyclonal rabbit anti-rat Fli-1 (cat no. ab133485) were obtained from Abcam (Cambridge, UK), extracellular signal-regulated kinase (ERK; cat. no. 9102) and phospho-ERK (cat. no. 9101) antibodies were obtained from Cell Signaling Technology, Inc. (CST; Danvers, MA, USA), whereas the antibody against GAPDH (cat. no. G9545) was purchased from Sigma-Alrich. All antibodies were used at a dilution of 1:1,000.

Reverse transcription-quantitative polymerase chain reaction ( $R T-q P C R)$. RNA was prepared by using TRIzol (cat. no. 155960181; Invitrogen; Thermo Fisher Scientific, Waltham, MA, USA) and cDNA was prepared by using a PrimeScript $^{\mathrm{TM}}$ RT reagent kit (cat. no. RR047A; Takara Bio, Inc., Otsu, Japan), according to the manufacturer's protocol. The $\beta$-actin gene was used to normalize the expression level; SYBR ${ }^{\circledR}$ Select Master mix reagent (cat. no. 4472908; 
Invitrogen; Thermo Fisher Scientific) was used for detection using the $2^{-\triangle \Delta C q}$ method (9). The primers for GATA1 amplification were: Sense, 5'-TGGTGGCTTTATGGTGGTG-3', anti-sense, 5'-CCTTGGTAGAGATGGGCAGT'-3; and the primers for $\beta$-actin amplification were: Sense, 5'-CATGTA CGTTGCTATCCAGGC-3', anti-sense, 5'-CTCCTTAATGTC ACGCACGAT-3'.

Luciferase reporter assay. HEK293T cells, plated in triplicate, were transfected with the indicated amounts of DNA (1 $\mu \mathrm{g}$ of pGL3Fli-1-BS using Lipofectamine ${ }^{\mathrm{TM}} 2000$ (Life Technologies; Thermo Fisher Scientific, Inc., Beijing, China) following the manufacturer's protocol. After $48 \mathrm{~h}$ of transfection, luciferase assays were performed in triplicate, as previously described (7).

Survival and statistical analysis. Mice survival rates were computed and plotted according to the nonparametric Kaplan-Meier analysis. Statistical analysis was performed using the two-tailed Student's $t$-test with analysis of variance, using Origin 3.5 software (Microcal Software, Northampton, MA, USA). $\mathrm{P}<0.05$ was considered to indicate a statistically significant difference.

\section{Results}

Anti-cancer activity of 150 TCM drug formulations on various cancer cell lines. TCM drug formulations are widely used in China for the treatment of various diseases with or without doctors' prescriptions. The present study aimed to examine the effect of $150 \mathrm{TCM}$ drugs used without drug prescription for anti-cancer activities. It was hypothesized that a novel anti-cancer TCM might be able to be administered in the clinic with fewer regulatory requirements. These drugs were dissolved in DMSO, and added to proliferating cultures of several cancer cell lines.

A drug, designated C54, was found to inhibit proliferation of the cell lines CB7, HEL and K562 (erythroleukemias), PC3 (prostate cancer), WM9 (melanoma) and MDA-MB-231 (breast cancer), with $\mathrm{IC}_{50}$ values in the range of 0.07-4.2 $\mu \mathrm{M}$ (Table I). The $\mathrm{IC}_{50}$ values were determined to be higher in the PC3, MDA-MB-231 and WM9 cell lines, which are known to have drug-resistant properties (10-12). C54 is a TCM formulation prescribed as pills ('Hou-Tong-Jie-Du-Wan') for the treatment of sore throats. It contains Realgar ( $\alpha$-As4S4, an arsenic sulfide mineral), Bos taurus domestius Gmelin (dried bovine gallstones), Borneol (a terpene) and cinobufagin venom toad organic material (from toad gland extract). Notably, Realgar and Borneol have been reported to have anti-cancer activity in various cancer cell lines $(13,14)$. Additionally, Borneol acts as a drug absorber (15), and Bos taurus domesticus Gmelin has been used to remove toxins from the body (see http://old. tcmwiki.com/wiki/calculus-bovis).

Subsequently, the mechanism of growth inhibition in the cell lines HEL, K562 and CB7, which exhibited the highest growth suppression activity with the C54 drug, was examined. Growth arrest in these cell lines in culture was revealed to be predominantly due to $G_{1}$ cell cycle arrest and the induction of apoptosis $24 \mathrm{~h}$ post-treatment (Fig. 1). HEL and CB7 cells expressed high levels of the Fli-1 oncogene (Fig. 2A and B).Fli-1 is a critical player
Table I. Determination of the $\mathrm{IC}_{50}$ values for various cancer cell lines with C54.

\begin{tabular}{lc}
\hline Cell line & $\mathrm{IC}_{50}(\mu \mathrm{M})$ \\
\hline HEL & $0.081 \pm 0.008$ \\
K562 & $0.073 \pm 0.001$ \\
CB7 & $0.573 \pm 0.014$ \\
MDA-MB-231 & $4.20 \pm 0.05$ \\
WM9 & $1.17 \pm 0.024$ \\
PC3 & $1.79 \pm 0.045$
\end{tabular}

Cells were treated with a series of drug doses of C54 for 3 days, and the data obtained using an MTT assay were used for calculating the $\mathrm{IC}_{50}$ values. $\mathrm{IC}_{50}$, concentration of the drug required to give halfmaximal inhibition.

in the induction and progression of F-MuLV-induced erythroleukemia (6,16-18). Notably, C54 markedly downregulated the expression of Fli-1 in HEL and CB7 cells, as determined by western blot analysis (Fig. 2A and B). In addition, in HEK293T cells transiently transfected with the Fli-1 expression vector, C54 inhibited the promoter activity of a PGL3-luciferase gene, driven by a minimum promoter and two Fli-1 binding sites, as previously described (Fig. 2C) (6). As anticipated, C54 upregulated the transcription of GATA1, a downstream target of Fli-1 whose expression is negatively regulated by this transcription factor (Fig. 2D) (19). C54 also downregulated the phosphorylation of mitogen-activated protein kinase (MAPK)/ERK in the HEL and CB7 cell lines (Fig. 2E and F). This growth signaling pathway is known to be activated by Fli-1 overexpression in leukemic cells $(20,21)$. These data, therefore, demonstrate that C54 acts as a potent inhibitor of Fli-1 expression and activity in leukemic cells.

Anti-cancer activity of the TCM drug, C54, on leukemia and breast cancer progression. To examine the anti-cancer activity of C54 in vivo, the mouse model of F-MuLV-induced erythroleukemia was used, in which Fli-1 activation through retroviral insertional mutagenesis induces erythroleukemias $(17,22)$. Treatment of mice with F-MuLV-induced erythroleukemias with $50 \mathrm{mg} / \mathrm{kg} \mathrm{C} 54$ resulted in no marked increase in leukemia-free survival (Fig. 3A). C54 resulted in a small, but significant reduction in the size of the spleen, the major site of tumor infiltration, when compared with vehicle-treated mice (Fig. 3B). Virus-induced leukemic mice develop anemia, which becomes severe as a result of tumor growth $(6,17)$. Although the control group of DMSO-treated leukemic mice developed massive anemia, C54-treated mice exhibited significantly increased hematocrit values, indicating therapeutic improvements and a slowing down of disease progression (Fig. 3C). These data suggested that, although C54 markedly inhibited Fli-1 in leukemic cells in vitro, it only exerted moderate anti-cancer effects in vivo.

Subsequently, the effect of C54 on breast cancer progression in an animal model of MDA-MB-231 cells orthotopically transplanted into the mammary fat pad of SCID mice was examined. In this experiment, C54 did not 
A

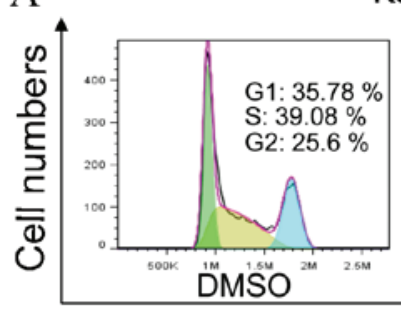

K562

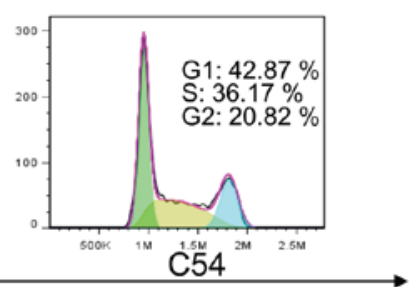

PI

CB7
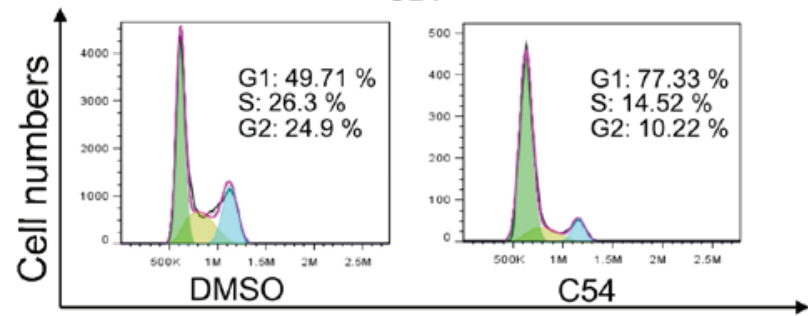

$\mathrm{Pl}$

HEL

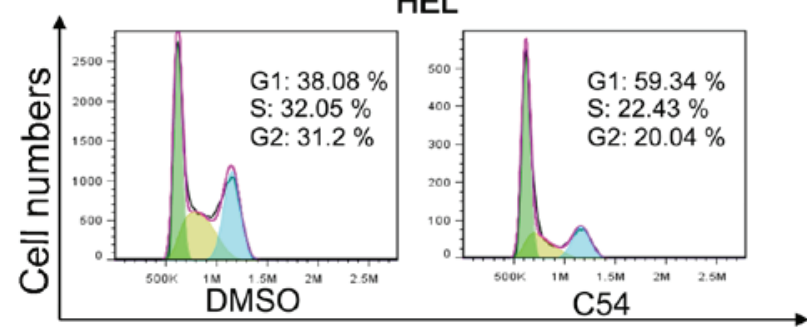

PI
B

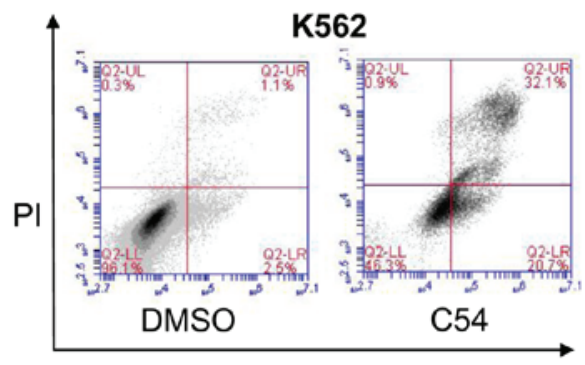

Annexin $\mathrm{V}$

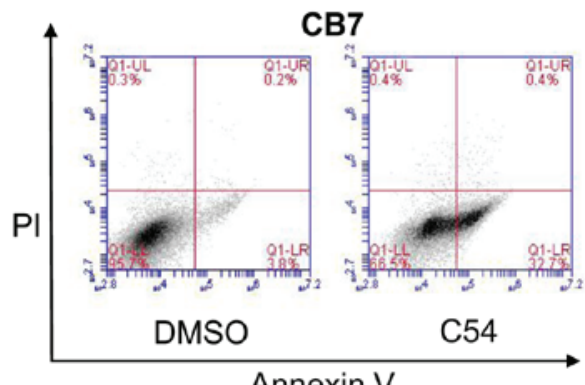

Annexin $V$

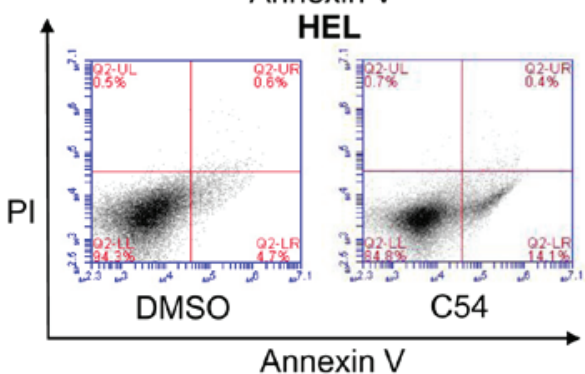

Figure 1. C54 induces apoptosis and increases $\mathrm{G}_{1}$ content in leukemic cells in culture. (A) C54-treated erythroleukemia lines K562, HEL and CB7 cells were subjected to cell-cycle analysis using PI flow cytometry. (B) Cells were cultured for $24 \mathrm{~h}$ in the presence of $5 \mu \mathrm{g} / \mathrm{ml} \mathrm{C} 54$, and stained using PI and Annexin V, as previously described (7). PI, propidium iodide; DMSO, dimethylsulfoxide.

A

DMSO

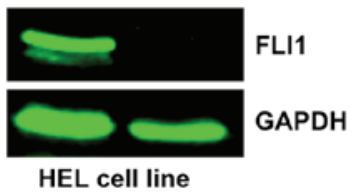

HEL cell line

B

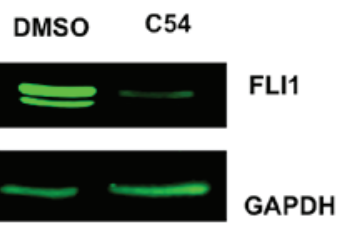

CB7 cell line
C

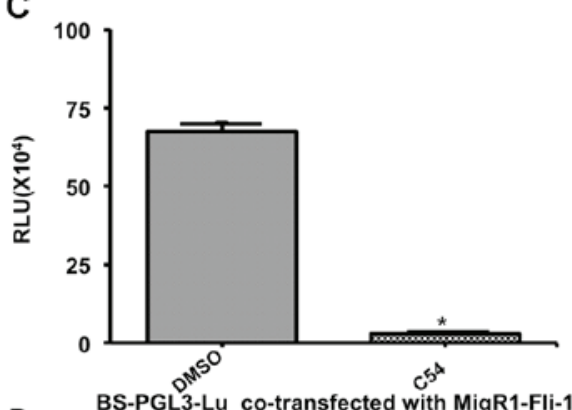

D

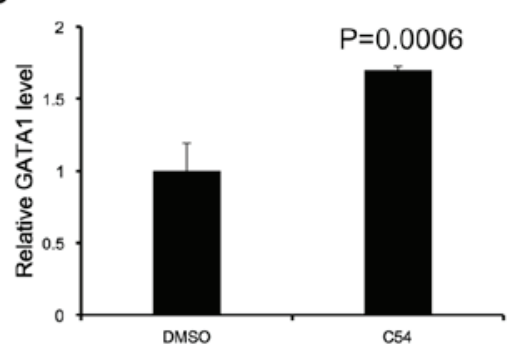

E

DMSO $\quad$ C54

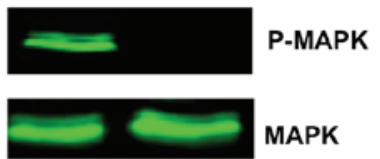

HEL cell line

MAPK
$\mathbf{F}$

DMSO $\quad$ C54

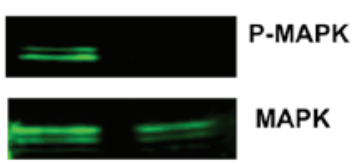

CB7 cell line

Figure 2. C54 inhibits expression and function of the Fli-1 oncogene in erythroleukemia cell lines. HEL (A) and CB7 (B) cells were cultured in the presence of $5 \mu \mathrm{g} / \mathrm{ml} \mathrm{C} 54$ for $12 \mathrm{~h}$, and then subjected to western blot analysis, as previously described (7). (C) 293T cells were transfected with a Fli-1 expression reporter (MigR1-Fli-1) and Fli-1-binding site promoter plasmid (BS-PGL3-Lu). As a control, the empty vector, MigR1, was transfected with BS-PGL3-Lu into 293T cells. After 48 h, cells were lysed and subjected to luciferase assays, as described previously (7). (D) HEL cells were incubated with C54 for $24 \mathrm{~h}$, and then RNA was isolated and subjected to RT-qPCR analysis using Fli-1 and control $\beta$-actin primers. (E) HEL cells were incubated with C54 for 24 h, proteins were isolated and subjected to western blot analysis using a Fli-1 antibody. $\beta$-actin was used as the loading control. (F) CB7 cells were treated with C54 for $24 \mathrm{~h}$ and subjected to western blot analysis using MAPK and P-MAPK antibodies. MAPK, mitogen-activated protein kinase; P-MAPK, phosphorylated MAPK; DMSO, dimethylsulfoxide; RLU, relative luciferase units. 


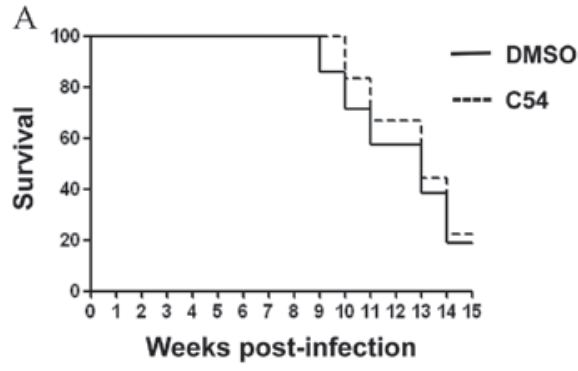

C

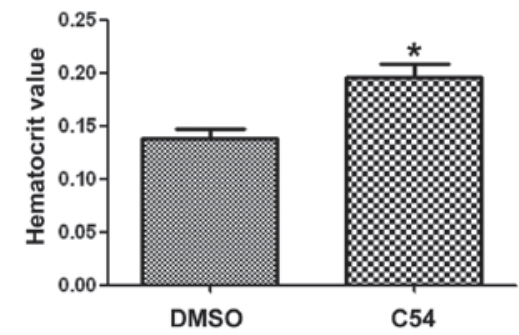

B

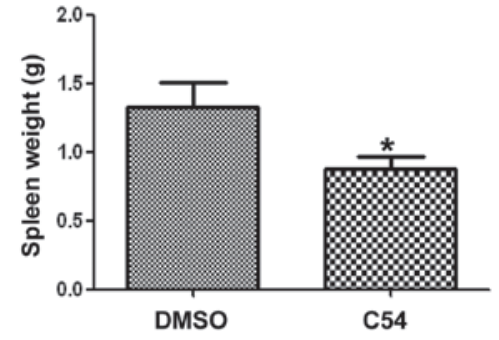

D

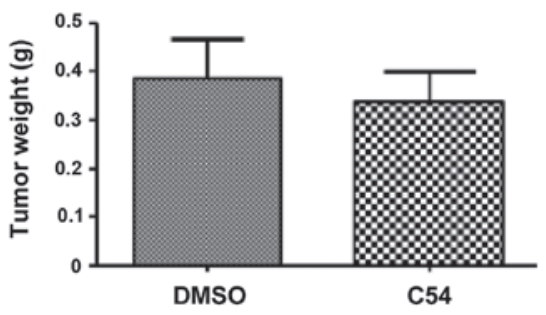

Figure 3. C54 does not significantly inhibit the progression of leukemia and breast cancer in mice. A group of 6-week-old mice ( $\mathrm{n}=6)$, infected at birth with F-MuLV, were treated every other day with C54 $(50 \mathrm{mg} / \mathrm{kg}$ of body weight) via IP injection for a period of 2 weeks. At the end points, when mice showed signs of morbidity from severe leukemia, animals were sacrificed by cervical dislocation and used to determine the survival rate (A), spleen weight (B) and hematocrit value (C). Note that treatment with C54 did not significantly delay leukemic development, manifested in an increased hematocrit value and reduced spleen size compared with the DMSO-injected control group. The statistical significance (P-value) was calculated using two-tail Student's $t$-tests. (D) A group of SCID mice $(n=6)$ were anesthetized with $10 \%$ chloral hydrate and injected orthotopically with MDA-MB-231 cells $\left(2 \times 10^{6}\right)$. After the tumors had reached $0.5 \mathrm{~cm}$ in diameter, mice were treated with C54 $(100 \mathrm{mg} / \mathrm{kg})$ every other day for 2 weeks via gavage. At 2 weeks following the drug treatment, mice were sacrificed and tumor weights were measured. No significant delays in tumor growth were observed. ${ }^{*} \mathrm{P}<0.05$ compared with the DMSO-treatment group. DMSO, dimethylsulfoxid; IP, intraperitoneal; F-MuLV, Friend murine leukemia virus; SCID, severe combined immunodeficiency.

A

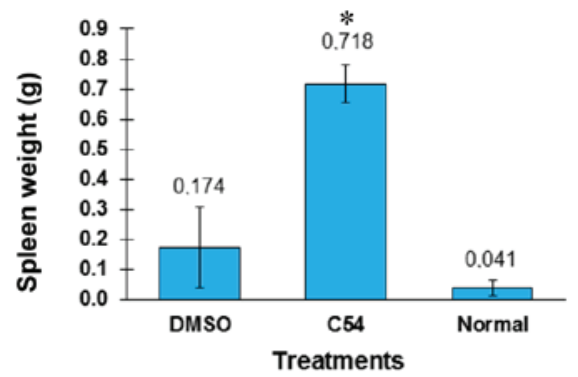

B

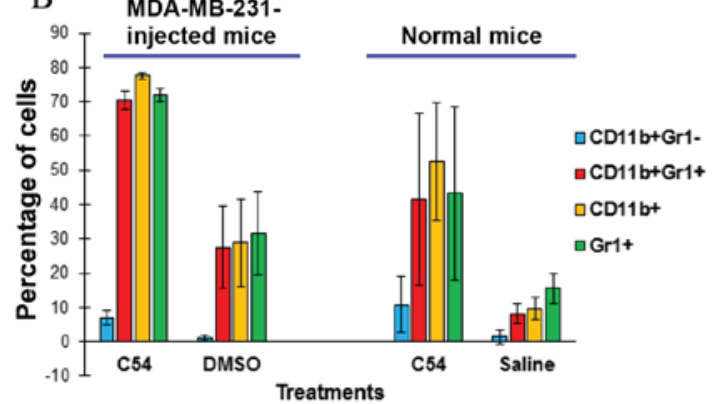

$\mathrm{C}$

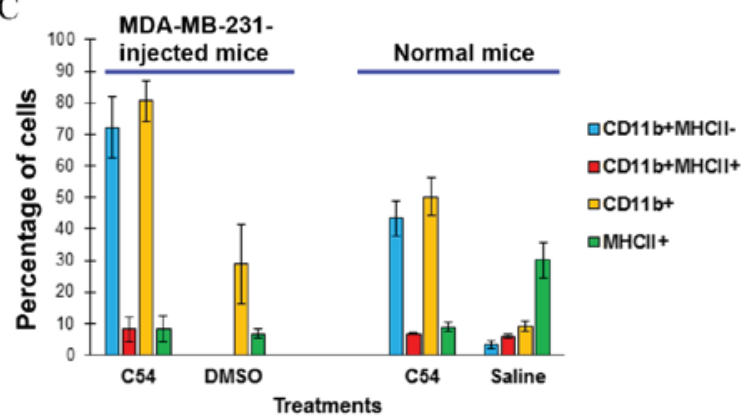

Figure 4. Treatment of mice with C54 induces inflammation associated with infiltration of suppressor myeloid cells in tumors and spleens. (A) SCID mice injected with MDA-MB-231 and treated with C54 developed larger spleens compared with the DMSO-treated mice. Spleens of normal SCID mice served as a control. A higher percentage of splenocytes isolated from tumor-bearing mice treated with C54 were revealed to be myeloid cells with expression

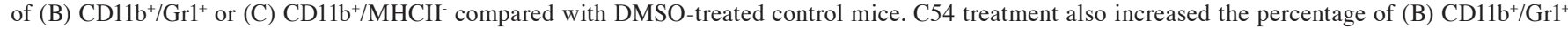

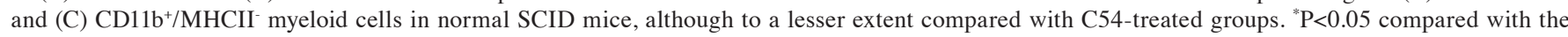
DMSO-treatment group. DMSO, dimethylsulfoxide; SCID, severe combined immunodeficiency; MHCII, major histocompatibility complex class II.

inhibit the growth of tumors in SCID mice (Fig. 3D). It was observed that, while the spleens of DMSO-treated leukemic mice were much larger compared with those of control SCID mice, the C54-treated leukemic group revealed an even more marked splenomegaly (Fig. 4A). Enlargement of the spleen was not due to tumor infiltration, as culturing splenocytes did 
not result in re-establishment of the MDA-MB-231 cell line (data not shown). Splenomegaly induced in immunodeficient SCID mice following the injection of MDA-MB-231 had been previously reported (23), although the underlying mechanism had not been elucidated. Notably, our findings revealed that injection of MDA-MB-231 increased the number of $\mathrm{CD}_{11} \mathrm{~b}^{+} / \mathrm{Gr}^{+}$monocytes in spleens of DMSO-treated mice, and that spleen size and the $\mathrm{CD} 11 \mathrm{~b}^{+} / \mathrm{Grl}^{+}$monocyte population further increased in C54-treated mice (Fig. 4B). CD11b ${ }^{+} / \mathrm{Gr}^{+}$ monocytes contribute to inflammation, which, in turn, is capable of supporting cancer progression through inflammatory factors (24). Normal BALB/c mice treated with C54 also developed splenomegaly, characterized by a higher infiltration by $\mathrm{CD} 11 \mathrm{~b}^{+} / \mathrm{Gr}^{+}$monocytes compared with controls (Fig. 4B). The CD1 $1 b^{+}$-positive splenocytes isolated from C54-treated, tumor-bearing or normal mice were predominantly negative for the expression of the MHCII antigen, which is mostly found on the surface of mature macrophages (Fig. 4C) (25).

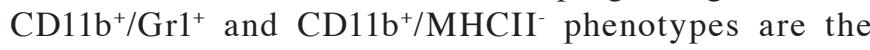
characteristic features of myeloid-derived suppressor cells (MDSCs), which are known to inhibit the immune system and accelerate tumor progression $(25,26)$. Taken together, these data have demonstrated that the induction of inflammation by C54 may outweigh its potent cell toxicity, thereby limiting its anti-cancer activity in vivo.

\section{Discussion}

In the past decade, increasing attention has been given to TCMs to satisfy the public's growing demands for alternative medicine. This has resulted in an acceleration in drug development and production in China and Western countries. In our laboratory, 150 TCM formulas were screened, which are used for various diseases in China in view of their anti-cancer activity: A biological activity that was not the principal purpose of the majority of these drugs. Among these, it has been revealed that the drug, $\mathrm{C} 54$, exhibited potent toxicity in all cancer cell lines tested in vitro. Despite this marked anti-cancer activity in vitro, C54 only moderately inhibited leukemogenesis in vivo, and did not exert any effect on the progression of transplanted human breast cancer in mice. To understand the underlying mechanism of this failed tumor inhibition, a number of experiments were designed, and it has been demonstrated that C54 may induce prompt inflammation in mice, leading to an accumulation of $\mathrm{CD} 11 \mathrm{~b}^{+} / \mathrm{Gr}^{+} \mathrm{MSDCs}$ in tumor masses and in the spleen of tumor-bearing, as well as normal, mice. These results indicate that the presence of both anti- and pro-tumorigenic compounds within C54 may hamper the potential of this drug to be used as an anti-cancer medicine for the treatment of various malignancies.

The anti-cancer activity of C54 is likely to be induced by its components, arsenic sulfide (Realgar) and Borneol, which are known to induce apoptosis in several cancer cell lines $(13,14)$. In China, Realgar and Borneol have been incorporated into many TCM formulas over a long period, and are considered to be safe, albeit with a few side-effects. Realgar appears to be the active ingredient of the Angong Niuhuang pill, used for protection against lipopolysaccharide (LPS)-induced neuroinflammation (27). Realgar with Cinnabar is also found in Wan-Sheng-Hua-Feng-Dan formulas, which have been demonstrated to protect against LPS-induced neurotoxicity (28). A similar anti-inflammatory capacity was recently reported for Borneol (29). The anti-inflammatory effects of Realgar and Borneol are primarily attributed to their ability to inhibit inflammatory factors $(28,29)$. It is noteworthy that two other components of C54, Bos taurus domestius Gmelin and cinobufagin venom toad, are also commercially being sold on the basis of their anti-inflammatory action. Thus, further research is required to identify the cause of inflammation by C54.

Treatment of erythroleukemic cells with C54 resulted in apoptosis, associated with a marked dowregulation of Fli-1. As Fli-1 expression serves an important role in the survival of erythroleukemic cells, and Fli-1 is known to be expressed in various leukemic cells, C54 represents a powerful drug for the treatment of hematological malignancies $(16,30)$. Thus, administration of C54 with a powerful anti-inflammatory drug could be a promising approach for the treatment of Fli-1-overexpressing leukemias. In support of this premise, our group has previously demonstrated that F-MuLV-induced erythroleukemogenesis was significantly inhibited when cancer-bearing mice were treated with either an anti-Fli-1 compound or an anti-inflammatory cyclo-oxygenase- 2 inhibitor, Celebrex (6,31).

Myeloid cells normally undergo differentiation to become granulocytes (neutrophils, basophils, eosinophils), macrophages and dendritic cells. However, under chronic inflammatory conditions, myeloid differentiation is skewed towards the expansion of MDSCs $(24,25)$. The suppressor function of MDSCs lies in their ability to inhibit adaptive and innate immune responses $(32,33)$. MDSCs are also known to secrete factors that are able to stimulate tumor growth through increased angiogenesis and metastasis $(32,33)$. In the mouse, MDSCs were phenotypically characterized as expressing high levels of CD11b (a classical myeloid lineage marker) and Gr1 (a granulocytic marker). The presence of higher numbers of MDSCs in tumor-bearing mice treated with C54 is likely to be the cause of failed tumor inhibition by this drug. These MDSCs are able to exert their tumor-promoting activity in normal and immunodeficient SCID mice. Thus, targeting MDSCs should strengthen the tumor-inhibiting ability of C54, leading to a slowing down of cancer.

Although C54 is not administered in the clinic for cancer treatment, this drug is being sold in China for the treatment of symptoms associated with having a cold and a sore throat. In this situation, this drug may exert its anti-viral and anti-bacterial activities through cell cytotoxicity and the induction of inflammation, which is beneficial for the patient in terms of curing the infectious disease. However, we propose that patients diagnosed with cancer should not take C54 to treat symptoms of cold, since the drug could worsen the cancer status.

In conclusion, we have identified a TCM drug with marked cytotoxicity in culture, although with limited or negligible tumor inhibitory activity in mice. This drug was shown to inhibit the expression and function of the oncogene Fli-1, involved in leukemogenesis. In addition, C54 enriched inflammatory blood monocytes in a cancer environment-a phenomenon that is known to accelerate cancer progression. Therefore, the anti-cancer activity of this formulation may 
potentially be improved when combined with an anti-inflammatory drug.

\section{Acknowledgements}

The present study was supported by research grants from the Science and Technology Department of Guizhou Province innovation and project grant (2013-6012), Thousand Talent Program of China (WQ20135200171) and The Natural Science Foundation of China (no. 81472609) to Y.B.D.

\section{References}

1. Shaw D: Toxicological risks of Chinese herbs. Planta Med 76 2012-2018, 2010

2. Wu XY, Tang JL, Mao C, Yuan JQ, Qin Y and Chung VC: Systematic reviews and meta-analyses of traditional chinese medicine must search chinese databases to reduce language bias. Evid Based Complement Alternat Med 2013: 812179, 2013.

3. Leung AY: Traditional toxicity documentation of Chinese Materia Medica-an overview. Toxicol Pathol 34: 319-326, 2006.

4. Yang JX and Wang XM: Progress in studies on anti-hepatoma effect of traditional Chinese medicine by adjusting immune function. Zhongguo Zhong Yao Za Zhi 32: 281-284, 2007 (In Chinese).

5. Yang D and Tian G: Review of experimental study on treatment of lung cancer with traditional Chinese medicine. Zhongguo Zhong Yao Za Zhi 34: 2405-2409, 2009 (In Chinese).

6. Li YJ, Zhao X, Vecchiarelli-Federico LM, Li Y, Datti A, Cheng Y and Ben-David Y: Drug-mediated inhibition of Fli-1 for the treatment of leukemia. Blood Cancer J 2: e54, 2012.

7. Li YJ, Liu G, Xia L, Xiao X, Liu JC, Menezes ME, Das SK, Emdad L, Sarkar D, Fisher PB, et al: Suppression of Her2/Neu mammary tumor development in mda-7/IL-24 transgenic mice. Oncotarget 6: 36943-36954, 2015.

8. Usenko T, Li YJ, Haeri M, Li Y, Vecchiarelli-Federico LM, Zhao X, Prchal JT and Ben-David Y: Enrichment of Sca1+ hematopoietic progenitors in polycythemic mice inhibits leukemogenesis. Blood 114: 1831-1841, 2009.

9. Livak KJ and Schmittgen TD: Analysis of Relative gene expression data using real-time quantitative PCR and the 2(-Delta Delta C(T)) Method. METHODS 25: 402-408, 2001.

10. Hermawan A, Wagner E and Roidl A: Consecutive salinomycin treatment reduces doxorubicin resistance of breast tumor cells by diminishing drug efflux pump expression and activity. Oncol Rep 35: 1732-1740, 2016

11. Yu X, Yang L, Cairns MJ, Dass C, Saravolac E, Li X and Sun LQ: Chemosensitization of solid tumors by inhibition of Bcl-xL expression using DNAzyme. Oncotarget 5: 9039-9048, 2014.

12. Lu SJ, Man S, Bani MR, Adachi D, Hawley RG, Kerbel RS and Ben-David Y: Retroviral insertional mutagenesis as a strategy for the identification of genes associated with cis-diamminedichloro platinum(II) resistance. Cancer Res 55: 1139-1145, 1995.

13. Pastorek M, Gronesova P, Cholujova D, Hunakova L, Bujnakova Z, Balaz P, Duraj J, Lee TC and Sedlak J: Realgar (As4S4) nanoparticles and arsenic trioxide (As2O3) induced autophagy and apoptosis in human melanoma cells in vitro. Neoplasma 61: 700-709, 2014.

14. Chen J, Li L, Su J, Li B, Chen T and Wong YS: Synergistic apoptosis-inducing effects on A375 human melanoma cells of natural borneol and curcumin. PLoS One 9: e101277, 2014.

15. Lu Y, Chen X, Du S, Wu Q, Yao Z and Zhai Y: The in situ and in vivo study on enhancing effect of borneol in nasal absorption of Geniposide in rats. Arch Pharm Res 33: 691-696, 2010.

16. Li Y, Luo H, Liu T, Zacksenhaus E and Ben-David Y: The ets transcription factor Fli-1 in development, cancer and disease. Oncotarget 34: 2022-2031, 2015.
17. Ben-David Y, Giddens EB, Letwin $\mathrm{K}$ and Bernstein A: Erythroleukemia induction by Friend murine leukemia virus: Insertional activation of a new member of the ets gene family, Fli-1, closely linked to c-ets-1. Genes Dev 5: 908-918, 1991.

18. Lee CR, Cervi D, Truong AH, Li YJ, Sarkar A and Ben-David Y Friend virus-induced erythroleukemias: A unique and well-defined mouse model for the development of leukemia. Anticancer Res 23: 2159-2166, 2003.

19. Athanasiou M, Mavrothalassitis G, Sun-Hoffman L and Blair DG: FLI-1 is a suppressor of erythroid differentiation in human hematopoietic cells. Leukemia 14: 439-445, 2000.

20. Zochodne B, Truong AH, Stetler K, Higgins RR, Howard J, Dumont D, Berger SA and Ben-David Y: Epo regulates erythroid proliferation and differentiation through distinct signaling pathways: Implication for erythropoiesis and Friend virus-induced erythroleukemia. Oncogene 19: 2296-2304, 2000.

21. Lakhanpal GK, Vecchiarelli-Federico LM, Li YJ, Cui JW, Bailey ML, Spaner DE, Dumont DJ, Barber DL and Ben-David Y: The inositol phosphatase SHIP-1 is negatively regulated by Fli-1 and its loss accelerates leukemogenesis. Blood 116: 428-436, 2010.

22. Ben-David Y, Giddens EB and Bernstein A: Identification and mapping of a common proviral integration site Fli-1 in erythroleukemia cells induced by Friend murine leukemia virus. Proc Natl Acad Sci USA 87: 1332-1336, 1990.

23. Emi M, Kim R, Tanabe K, Uchida Y and Toge T: Targeted therapy against $\mathrm{Bcl}-2$-related proteins in breast cancer cells. Breast Cancer Res 7: R940-952, 2005.

24. Nold MF, Mangan NE, Rudloff I, Cho SX, Shariatian N, Samarasinghe TD, Skuza EM, Pedersen J, Veldman A, Berger PJ and Nold-Petry CA: Interleukin-1 receptor antagonist prevents murine bronchopulmonary dysplasia induced by perinatal inflammation and hyperoxia. Proc Natl Acad Sci USA 110: 14384-14389, 2013.

25. Gabrilovich DI and Nagaraj S: Myeloid-derived suppressor cells as regulators of the immune system. Nat Rev Immunol 9: 162-174, 2009.

26. Meyer C, Sevko A, Ramacher M, Bazhin AV, Falk CS, Osen W, Borrello I, Kato M, Schadendorf D, Baniyash M and Umansky V: Chronic inflammation promotes myeloid-derived suppressor cell activation blocking antitumor immunity in transgenic mouse melanoma model. Proc Natl Acad Sci USA 108: 17111-17116, 2011.

27. Zhang F, Lu Y, Liu J and Shi J: Realgar is active ingredient of Angong Niuhuang pill in protection against LPS-induced neuroinflammation. Zhongguo Zhong Yao Za Zhi 35: 3333-3338, 2010 (In Chinese)

28. Zhang F, Lu Y, Wu Q, Yan J, Shi J and Liu J: Role of cinnabar and realgar of WSHFD in protecting against LPS-induced neurotoxicity. J Ethnopharmacol 139: 822-828, 2012

29. Zhong W, Cui Y, Yu Q, Xie X, Liu Y, Wei M, Ci X and Peng L: Modulation of LPS-stimulated pulmonary inflammation by Borneol in murine acute lung injury model. Inflammation 37: 1148-1157, 2014.

30. Cui JW, Vecchiarelli-Federico LM, Li YJ, Wang GJ and Ben-David Y: Continuous Fli-1 expression plays an essential role in the proliferation and survival of F-MuLV-induced erythroleukemia and human erythroleukemia. Leukemia 23: 1311-1319, 2009.

31. Cervi D, Klement G, Stempak D, Baruchel S, Koki A and Ben-David Y: Targeting cyclooxygenase-2 reduces overt toxicity toward low-dose vinblastine and extends survival of juvenile mice with Friend disease. Clin Cancer Res 11: 712-719, 2005.

32. Motallebnezhad M, Jadidi-Niaragh F, Qamsari ES, Bagheri S, Gharibi T and Yousefi M: The immunobiology of myeloid-derived suppressor cells in cancer. Tumour Biol 37: 1387-1406, 2016.

33. ParkerKH,Beury DW and Ostrand-Rosenberg S: Myeloid-derived suppressor cells: Critical cells driving immune suppression in the tumor microenvironment. Adv Cancer Res 128: 95-139, 2015. 